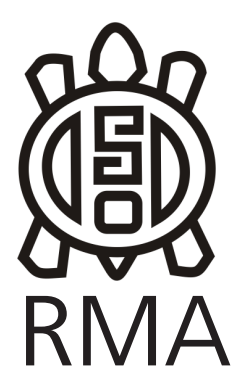

\title{
III Taller de Discusión sobre Restitución de Restos Humanos de Interés Arqueológico y Bioantropológico
}

\author{
María Luz Endere*, Gustavo Flensborg**, Mariela González***, Pablo \\ Bayala****, María Gabriela Chaparro****, Mónica Berón******, \\ Cristian Favier Dubois $* * * * * *$
}

*INCUAPA-CONICET-PATRIMONIA, Facultad de Ciencias Sociales, Universidad Nacional del Centro de la Provincia de Buenos

Aires, Olavarría, Argentina. mendere@soc.unicen.edu.ar. ${ }^{* *}$ INCUAPA-CONICET, Facultad de Ciencias Sociales, Universidad

Nacional del Centro de la Provincia de Buenos Aires. Olavarría, Argentina. gflensbo@soc.unicen.edu.ar. ${ }^{* * * I N C U A P A-}$

CONICET, Facultad de Ciencias Sociales, Universidad Nacional del Centro de la Provincia de Buenos Aires, Olavarría, Argentina.

mgonzalez@soc.unicen.edu.ar. ${ }^{* * *}$ INCUAPA-CONICET, Facultad de Ciencias Sociales, Universidad Nacional del Centro de la

Provincia de Buenos Aires, Olavarría, Argentina. pbayala@soc.unicen.edu.ar. *****INCUAPA-CONICET-PATRIMONIA, Facultad de

Ciencias Sociales, Universidad Nacional del Centro de la Provincia de Buenos Aires, Olavarría, Argentina.

chaparro@soc.unicen.edu.ar; *****INCUAPA-CONICET, Facultad de Ciencias Sociales, Universidad Nacional del Centro de la Provincia de Buenos Aires. Museo Etnográfico "Juan B. Ambrosetti", Universidad de Buenos Aires, Argentina. monberon@retina.ar; ${ }^{* * * * * * I N C U A P A-C O N I C E T, ~ F a c u l t a d ~ d e ~ C i e n c i a s ~ S o c i a l e s, ~ U n i v e r s i d a d ~ N a c i o n a l ~ d e l ~ C e n t r o ~ d e ~ l a ~ P r o v i n c i a ~}$ de Buenos Aires, Olavarría, Argentina. cfavier3@gmail.com.

\begin{abstract}
Resumen
En este trabajo se presentan los resultados del III Taller de Discusión sobre Restitución de Restos Humanos de Interés Arqueológico llevado a cabo en Junio de 2013 en la ciudad de Olavarría.
\end{abstract}

Palabras Clave: restos humanos de origen arqueológico, restitución, etica, práctica profesional.

III Discussion Workshop on the Restitution of Human Remains of Archaeological and Bioanthropological Interest

\begin{abstract} interest, held in June 2013 in the city of Olavarria.

Durante los días 13 y 14 de junio de 2013 se realizó en la ciudad de Olavarría, provincia de Buenos Aires, el III Taller de Discusión sobre Restitución de Restos Humanos de Interés Arqueológico y Bioantropológico (III TaDiRH ${ }^{1}$ ), co-organizado por el INCUAPACONICET-UNICEN y la Facultad de Ciencias Sociales de la UNICEN y auspiciado por la Sociedad Argentina de Antropología (SAA). Este taller es la continuación de dos encuentros que se realizaron en Buenos Aires y Mar del Plata en el año 2011, con los objetivos de informar a la comunidad académica acerca del alcance del Decreto 701/10 que reglamenta la Ley Nacional 25.517 y debatir sus implicancias en la práctica profesional.
\end{abstract}

This paper presents the results of the III Discussion Workshop to discuss human remains restitution of archaeological

Keywords: human remains from archaeological contexts, restitution, ethics, professional practice.

Desde la sanción del mencionado decreto, hace tres años, se cuenta con diferentes experiencias de aplicación del mismo y han surgido dificultades que hicieron necesario generar un espacio de discusión que reuniera a distintos investigadores e instituciones involucradas del país. Por tal motivo, en este encuentro se propuso debatir y tratar de arribar a consensos respecto de

${ }^{1}$ Esta reunión fue financiada con un subsidio especial otorgado por la Facultad de Ciencias Sociales y la SECAT-UNICEN. criterios y líneas de acción a adoptar entre profesionales de la Arqueología y la Bioantropología en relación al tratamiento ético de restos humanos, su restitución y a la instrumentación del protocolo de consentimiento previo, libre e informado de los pueblos originarios. Teniendo en cuenta estos objetivos, la convocatoria fue destinada, en esta instancia, a arqueólogos y antropólogos biólogos con el propósito de comentar experiencias, discutir propuestas sobre modos de proceder consensuados en la actividad profesional y, en especial, en relación con los pueblos originarios. Se considera que alcanzar algún grado de acuerdo en la comunidad académica facilitará el diálogo posterior con los distintos sectores sociales involucrados (e.g., pueblos originarios, comunidades locales). En este marco, las actividades del III TaDiRH consistieron en una charla introductoria a la temática desde el punto de vista legal y una presentación del estado de la cuestión y del grado de avance alcanzado en los talleres previos. Posteriormente, se abrieron tres mesas de discusión que funcionaron en forma consecutiva y que abordaron los siguientes temas: el protocolo de consentimiento previo, libre e informado, la restitución y colecciones arqueológicas, y los procedimientos y recaudos éticos. 
En esta reunión se contó con un número importante de participantes, totalizando 63 asistentes de distintos puntos del país, muchos de ellos en representación de diversas instituciones. En efecto, asistieron miembros del Museo Etnográfico J. B. Ambrosetti (UBA), Museo de La Plata (FCNyM, UNLP), Museo de Antropología de Córdoba (UNC), así como de asociaciones profesionales tales como la Sociedad Argentina de Antropología (SAA), la Asociación de Arqueólogos Profesionales de la República Argentina (AAPRA) y la Asociación de Antropología Biológica de Argentina (AABA). A esta lista se sumaron los directores y representantes de otras instituciones públicas, universidades, centros de investigación y laboratorios como el CENPATCONICET, CONICET (IMHICIHU), CEBBAD-CONICET, LEEH, INAPL, UNRC, UBA, UNC, UNLP, UNS, UNICEN, Secretaria de Cultura de la provincia de Jujuy, Universidad de Michigan (EEUU), e INCUAPA-CONICET. Esta numerosa y activa participación de los profesionales constituye un claro indicio del interés que existe por obtener información y conocer la legislación vigente, así como por atender a los reclamos de los pueblos originarios.

Como resultado final del plenario con el que concluyó la reunión, se elaboraron los siguientes puntos de acuerdo:

1. Manifestamos la necesidad de intensificar el debate sobre este tema entre investigadores y personal involucrado en el manejo de colecciones en los ámbitos profesionales, científicos y académicos. 2. Resaltamos la importancia de intensificar el diálogo intra e intercultural con los diferentes actores sociales con el objetivo de reflexionar y enriquecer la práctica profesional.

3. Acordamos la necesidad de hacer extensiva la discusión a todo el patrimonio cultural material e inmaterial y, en ese contexto, considerar las cuestiones relativas a la restitución de restos humanos.

4. Solicitamos la presencia efectiva del Estado Nacional y de los estados provinciales para respaldar los procedimientos profesionales en las distintas instancias de manejo de los materiales arqueológicos y bioantropológicos e impulsar la celebración de acuerdos entre los actores sociales involucrados. 5. Enfatizamos la necesidad de que los representantes de nuestras asociaciones profesionales estén presentes y tengan una participación activa en las reuniones del INAI en donde se traten los temas atenientes a la aplicación y puesta en marcha de la Ley 25.517.

6. Consideramos esencial hacer explícito el aporte de la arqueología, la antropología biológica, social y lingüística, así como justificar nuestro derecho a participar en las discusiones sobre la instrumentación del protocolo de consentimiento libre, previo e informado (CLPI) que se lleven a cabo por iniciativa del INAI, a fin de hacerlo viable en la práctica, en especial en relación con los plazos en los que se deban expedir los pueblos originarios y el idioma en que deba ser redactado dicho protocolo.

7. Ponemos de manifiesto el problema de identificar la contraparte legalmente válida a quien deba solicitársele el CLPI, teniendo en cuenta que coexisten con el registro del INAI otros registros provinciales, y que hay comunidades que no están registradas. 8. Manifestamos la importancia de contar con una participación activa de las diferentes instituciones involucradas. En este sentido, solicitamos a nuestras instituciones representativas (universidades, institutos, ANPCyT, CONICET, etc.) su intervención en esta cuestión, ya que ello puede afectar la iniciación y/o continuidad de nuestros proyectos de investigación.

9. Acordamos además sobre la necesidad de elaborar lineamientos generales para un protocolo profesional base de consentimiento informado, que se ajuste a las características del patrimonio cultural y de las investigaciones que se realizan sobre ellos, a fin de servir de orientación para futuras instrumentaciones.

9.1. En esta línea de pensamiento, consideramos que el consentimiento informado no consiste en un acto concreto sino en un proceso dinámico de entendimiento mutuo.

9.2. Acordamos que en dichos lineamientos deben definirse los ítems que debe contener el protocolo básico de consentimiento, incluyendo las cuestiones relativas a los restos humanos que son estudiados en el marco de investigaciones forenses.

10. Recomendamos contemplar en los proyectos de investigación las tareas y los plazos que implica establecer un diálogo con los pueblos indígenas y presupuestar fondos destinados a la conservación, documentación y puesta en valor del patrimonio recuperado.

11. Consideramos que los pedidos de restitución de restos humanos deberían ser tratados según las particularidades de cada caso.

12. Sugerimos especial cuidado respecto de la terminología utilizada para referirse a los restos humanos en publicaciones científicas.

13. Proponemos difundir ampliamente estas conclusiones en los ámbitos profesionales y académicos a fin de ampliar el debate en instancias futuras.

14. Deseamos transmitir estas conclusiones y hacer extensivo este debate a directivos responsables, profesionales y trabajadores de otras instituciones que posean colecciones de restos humanos de interés arqueológico y bioantropológico como Parques Nacionales, hospitales, museos, etc.

15. Aceptamos la moción de efectuar una reunión en el plazo de dos meses, convocada por la AAPRA, con representantes de diferentes instituciones a fin de elaborar el proyecto de protocolo base. Adhieren a esta convocatoria representantes de la SAA y de la $A A B A$ que participaron de este taller.

16. Acordamos realizar el IV TaDiRH en la Facultad de Ciencias Naturales y Museo (UNLP) en la ciudad de La Plata, en el mes de abril de 2014.

17. Acordamos crear un foro de discusión e intercambio de información entre los participantes de este taller.

A modo de conclusión puede afirmarse que este taller constituyó una interesante experiencia y resultó una instancia valiosa en el sentido que fue posible congregar a diferentes profesionales y discutir de manera fructífera acerca de los alcances del Decreto 701/10. De todos modos, tal como queda expresado en los puntos 15 y 16, aún resta seguir trabajando en este aspecto que tiene implicancias directas en la práctica profesional y en el modo de interactuar con los pueblos originarios, a fin de atender adecuadamente a sus reclamos.

Olavarría, 10 de septiembre de 2013 